This a postprint (paper accepted for publication, after peer review) of :

R. Mitteau, J.M. Missiaen, P. Brustolin, O. Ozer, A. Durocher, C. Ruset, C.P. Lungu, X.

Courtois, C. Dominicy, H. Maier, C. Grisolia, G. Piazza, P. Chappuis,

Recent developments toward the use of tungsten as armour material in plasma facing components,

Fusion Engineering and Design, Volume 82, Issues 15-24, 2007, Pages 1700-1705, ISSN 0920-3796,

https://doi.org/10.1016/j.fusengdes.2007.01.003

http://www.sciencedirect.com/science/article/pii/S0920379607000245

Some reference which were 'to be published' at the time of the writing of the paper have been updated.

\title{
RECENT DEVELOPMENTS TOWARD THE USE OF TUNGSTEN AS ARMOUR MATERIAL IN PLASMA FACING COMPONENTS
}

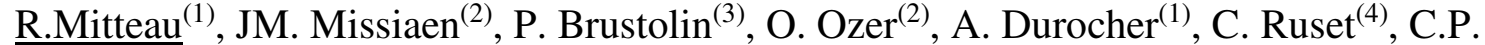

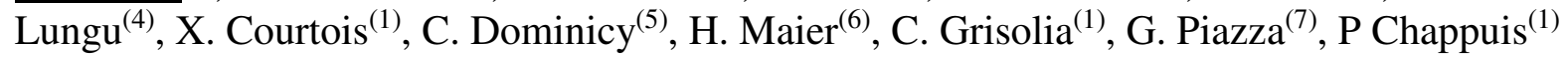 \\ ${ }^{(1)}$ Euratom - CEA, Direction des sciences de matière, département de recherches sur la fusion \\ contrôlée, CEA Cadarache, Saint Paul les Durances, France \\ (2) LTPCM, UMR CNRS 5614, Université Joseph Fourier, Grenoble, France \\ (3) Institut de Soudure, YUTZ, France \\ (4) NILPRP, Magurele-Bucharest, Romania \\ ${ }^{(5)}$ CP2M, Université d'Aix Marseille III, Marseille, France \\ ${ }^{(6)}$ Max-Planck-Institut für Plasmaphysik, Euratom Association, Garching, Germany \\ ${ }^{(7)}$ EFDA JET , Close Support Unit, Culham Science Centre, UK
}

\section{ABSTRACT}

Future fusion experiments will rely on tungsten armour tile for their plasma facing components. In order to sustain steady state operation, the components need to be cooled through an attachment to a heat sink. All current reference concepts rely on contact bonds, unfavourable for long term application (high temperature service, cycle fatigue, thermal shocks). Three routes toward the development of thick tungsten bonds are presented here, namely functionally graded tungsten copper assembly, electron beam welding of tungsten, and coating processes. All present favourable prospects, and tend to indicate that a thick bond is possible with tungsten. Dedicated programs as well as industrial implication are however required if such concepts are to be used actually for the fabrication of large components series.

\section{INTRODUCTION}

The tendency toward steady state fusion experiments imposes wear resistant armour materials to face the plasma and intimate bonding to cooled structures. The practical consequences for future plasma facing components (PFC) are multi-millimetre armour thicknesses and heterogeneous bonds, as the armour tiles are usually made out of a refractory material with a low thermal expansion coefficient [1]. At the same time, tungsten is increasingly considered as a prime candidate armour material facing the plasma in tokamaks for medium to high heat flux components (ASDEX, JET, ITER), essentially because of its low tritium retention property. This 
material is, however, a challenge for the engineers due to its hardness, its high fusion temperature, its brittleness at room temperature, and to its low recrystallisation temperature compared to the fusion temperature. Additionally it is difficult to manufacture by processes based on milling and welding. The current reference concepts for tungsten armoured plasma facing components all rely on contacts bonds (hot isostatic pressing, active metal casting, brazing), with typical cross - bond dimensions of less than $0.1 \mathrm{~mm}$ [2-4]. Test mock-ups have undergone successfully cycled high heat flux tests [5], however such bonds are still considered unfavourable regarding high temperature deformation, cycle fatigue, creep or thermal shocks [6,7]. Bonds with larger dimensions (more than $1 \mathrm{~mm}$ ) and a more intimate intermixing of the two materials would be more favourable. Many of the developments pursued at Tore Supra during the last decade aimed at a diffuse bond between the carbon fiber composite (CFC, the workhorse armour material a decade ago) which ended successfully with the development of the structured active metal casting ${ }^{\circledR}$ (AMC) at Plansee and to the manufacture of the toroidal pumped limiter. The recent shift toward tungsten led to try to transpose that experience to $\mathrm{W}$-heat sink bonds. Preliminary trials of diffuse bonds are promoted, through collaboration with various French and European research institutes. These are 1) functionally graded tungsten to copper, 2) direct electron beam welding of tungsten to Mo-alloy TZM and 3) the characterisation of tungsten coatings deposited on carbon fibre composite by high energy deposition processes. Elements on these working directions are given below.

\section{PROCESSING OF TUNGSTEN TO COPPER SOFT TRANSITION}

Functionally graded materials (FGM) eliminate the need for heterogeneous assembly, by having a smooth transition between the armour and the cooled substrate. The singular point at the free interface, where stresses concentrates and weaken the assembly, is eliminated, allowing a better behaviour. Thermomechanical damage during thermal processing is limited by the smooth composition variation and by the high creep strength of $\mathrm{W}-\mathrm{Cu}$ alloys. Among the several process routes that exists to manufacture FGM, powder metallurgy open the way for an integrated manufacturing route for first wall elements and divertor targets with graded structures. $\mathrm{W}-\mathrm{Cu}$ powder mixtures exhibit a poor sinterability, due to the weak chemical interaction between $\mathrm{W}$ and $\mathrm{Cu}$. Relatively high sintered densities could however be obtained recently by sintering $\mathrm{W}-\mathrm{CuO}$ powder mixtures under reducing atmosphere [9]. This was the starting point for the study presented here. In a first manufacturing route (Fig. 1), the thermal reduction of the copper oxide was carried out during the sintering, using a $\mathrm{He} / \mathrm{H}$ flow.

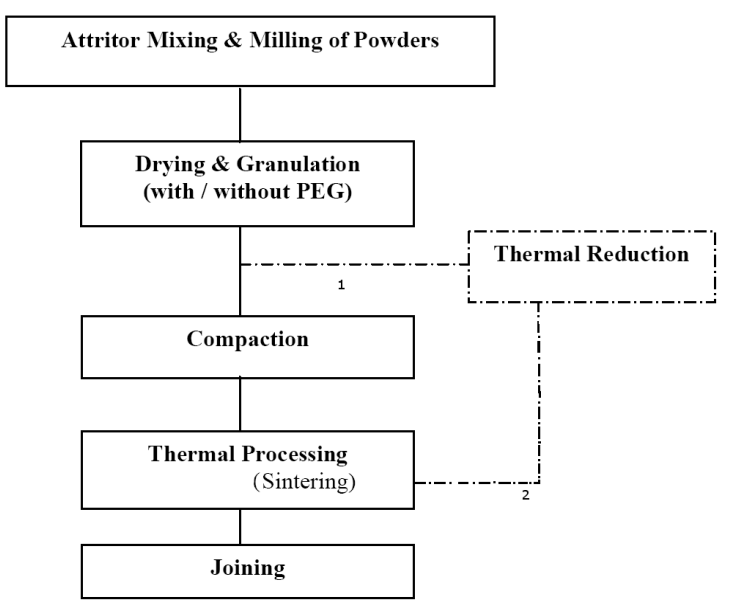

Figure 1 : Processing routes for FGM materials

Reduction steps were deduced from thermo-gravimetric analyses (TGA), and the sintering using dilatometry. Solid-state reaction between copper oxide and tungsten particles was first suspected by TGA, then evidenced by transmission electron microscope observation of tungsten oxides. More critical was the evidence of swelling effects associated to the $\mathrm{CuO}$ reduction steps, which were related both to the formation of porous $\mathrm{Cu}$ 
particles and to the formation of the tungsten oxides. Swelling effects limit the final density obtainable through the sintering step and could cause delamination during processing of gradient materials. For that reason, the manufacturing route was improved through a pre-reduction at $350^{\circ} \mathrm{C}$ during $2 \mathrm{~h}$ of the copper oxide powder (route 2 in Fig. 1). A high compaction efficiency and a good sinterability could then be obtained with density $92-95 \%$ of the theoretical density, and containing essentially closed pores. Dilatometric analyses of the three compositions looked promising since the shrinkage amplitude and temperature ranges are similar for the three compositions. Graded W-Cu $(10 / 20 / 30 \% \mathrm{Cu})$ could be realized by a cocompaction / co-firing process at $1300^{\circ} \mathrm{C}$, 2h [9]. Relatively dense microstructures could thus be obtained $(\approx 95 \%$ theoretical density) with porosity in the sintered material is essentially closed, and a good adhesion between the layers (Fig. 2, 3).

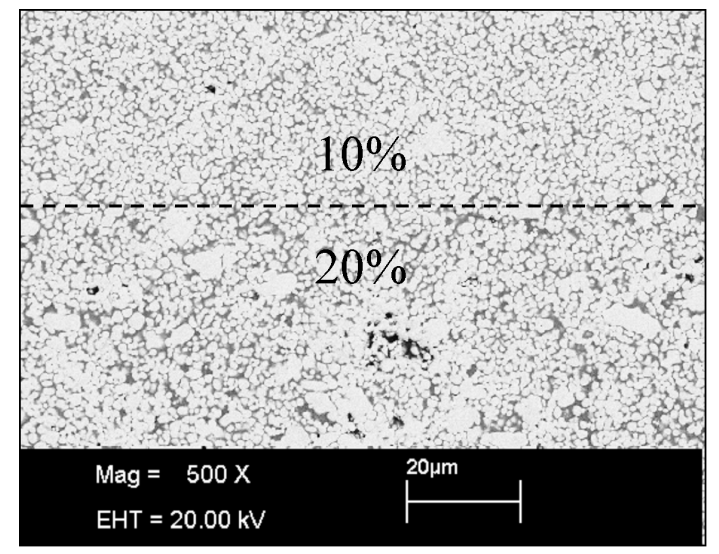

Figure 2 : Boundary layer between 10 $20 \% \mathrm{Cu}$ layers on microstructure of $\mathrm{W}-\mathrm{Cu}$.

However, an important problem in the processing of graded structures is liquid phase migration between the layers. This phenomenon is systematically observed, causes distortion of the parts, and considerably smoothes the composition gradient.

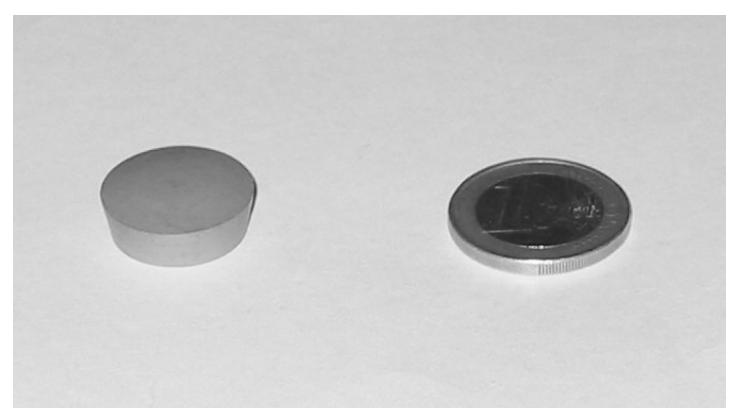

Figure 3 : View of a W-Cu $(10 / 20 \% \mathrm{Cu})$ graded part, sintered at $1300^{\circ} \mathrm{C}, 2 \mathrm{~h}$.

This problem should be solved in the future to benefit of the properties of the graded composition structure. Liquid migration can indeed be reduced by optimization of the microstructure of the layers (grain size, porosity...) as shown from recent models based on interface energy minimization. Future work aims also to improve the process by using spark or microwave assisted sintering.

\section{ELECTRON BEAM WELDING OF TUNGSTEN TO MO-ALLOY TZM}

This assembly is investigated to produce high temperature components required by radiation cooled PFCs [10]. The driving line is to use simple tungsten shapes for the tile, and to have the complex attachment part in TZM in order to reduce the milling cost (thus the use of TZM, a molybdenum alloy which is more practical and cheaper to mill than tungsten). Such components require no heat sink and there is no vacuum tightness function regarding a cooling medium, so that only good mechanical properties are required. The electron beam welding produces a well diffused bond, representative of what is aimed at. The welding tests have been performed on a $30 \mathrm{KW}$ electron beam test rig $(60 \mathrm{KV}, 500 \mathrm{~mA})$. The beam was vertical and the welding displacement horizontal. In spite of reputed low weldability properties of the refractory alloys, a good bonding up to a depth of 5 $\mathrm{mm}$ is obtained (Fig. 4). 


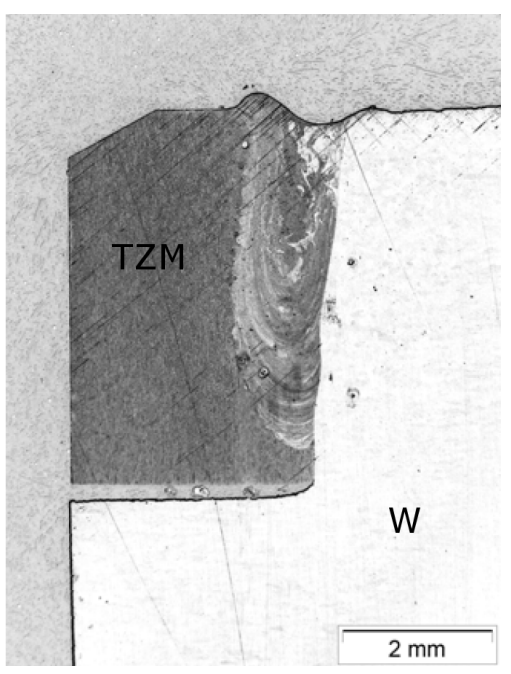

Figure 4 : Electron beam weld cross section

Tests have been performed with and without preheating, the former giving qualitatively better results. There are no apparent cracks. Some voids are present, and there are spherical which is admitted as tolerable in such welds. There is some evolution of the microstructure in the thermally affected zones (table 1), which undergo probably a recrystallisation. (associated to a texture reduction). Grain growth is present, but to a limited extent (less than $50 \%$ ). The microstructure is sound and representative of good quality welds. Hardness measurements (Vickers) have been performed, using a $20 \mathrm{~kg}$ weight (Fig. 5). The melt area and the heat affected zone are harder than TZM, the weakest materials at $230 \mathrm{Hv}$. There is no softening of the materials in the heat affected zone, so that it can be concluded that welding using electron beam technology bring no weak link between the two materials. Quench tests in water from up white heated samples $\left(>1000^{\circ} \mathrm{C}\right.$ ) are done without apparent crack formation. These very elementary tests confirm the weldabilty of $\mathrm{W}$ on TZM and make the testing of electron beam weld to other substrates very interesting.

\begin{tabular}{|c|c|c|c|c|c|c|}
\hline $\begin{array}{l}\text { micro- } \\
\text { graphs }\end{array}$ & rim & Hed & (1) & $y$ & & 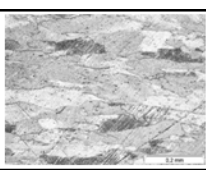 \\
\hline location & $\begin{array}{l}\text { Non } \\
\text { affected } \\
\text { bulk-W }\end{array}$ & $\begin{array}{l}\text { HAZ } \\
\text { (W-side) }\end{array}$ & $\begin{array}{l}\text { Melted } \\
\text { zone } \\
\text { (W-side) }\end{array}$ & $\begin{array}{l}\text { Melted area } \\
\text { (TZM-side) }\end{array}$ & $\begin{array}{l}\text { HAZ } \\
\text { (TZM-side) }\end{array}$ & $\begin{array}{l}\text { Non } \\
\text { affected } \\
\text { bulk TZM }\end{array}$ \\
\hline grain size & $100 \mu \mathrm{m}$ & $100 \mu \mathrm{m}$ & $150 \mu \mathrm{m}$ & $400 \mu \mathrm{m}$ & $150 \mu \mathrm{m}$ & $300 \mu \mathrm{m}$ \\
\hline texture & strong & moderate & moderate & equiaxe & equiaxe & strong \\
\hline
\end{tabular}

Table 1 : Weld microstructure of the at different locations (scale is identical on all photos)

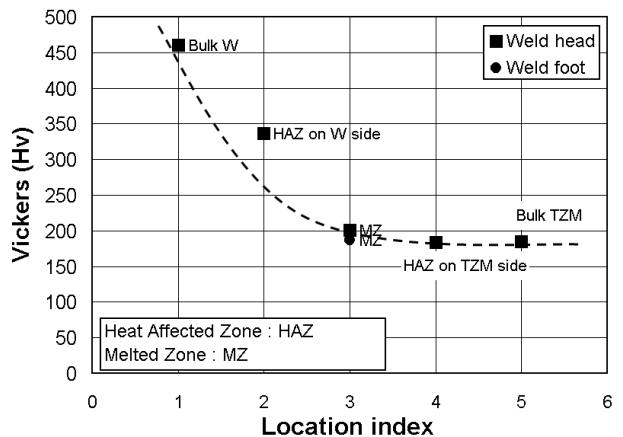

Figure 5 : Hardness profile through the weld

\section{TUNGSTEN COATING ON CARBON FIBER COMPOSITE}

This topic is practically not relevant for actively cooled plasma facing components, but the interest stems from the development of an interlayer between the CFC and the W. Two kinds of coatings are manufactured and optimised by EuratomRomania Association using Combined Magnetron Sputtering and Ion Implantation (CMSII, [11]) and Thermoionic Vacuum Arc (TVA, [12]). The analysis focuses on CMSII which produced better samples during the preliminary 
trials. Two samples (CMSII3 and CMSII4) were available for analysis. The coating morphology is characterised by a scanning electron microscope (Fig. 6). A wedge was cut thought the layers using a focused ion beam (FIB), allowing a very clean view of the cross section. No void is observed between the Mo interlayer and the tungsten, at the location of the FIB wedge for CSMII-3, although some localised delamination could be observed on CSMII4.

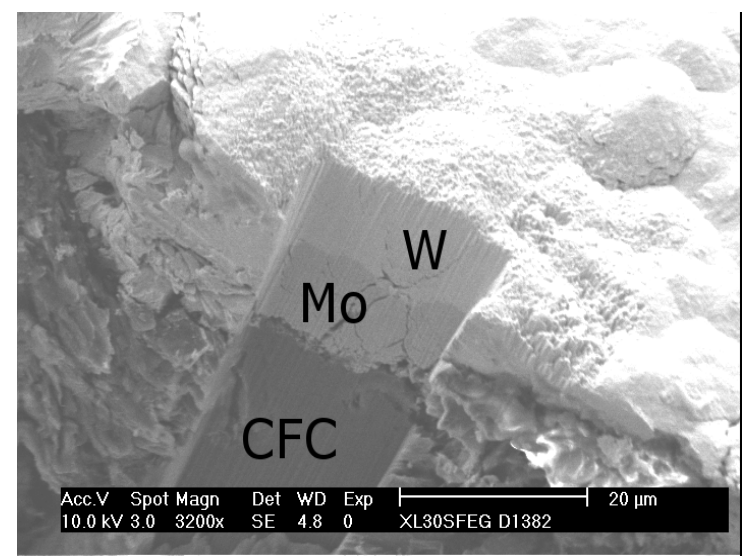

Figure 6 : Scanning electron microscope image of a wedge cut through the coating

As expected, large voids and crack are observed in the carbon fiber composite, but this tormented structure is well bridged by the coating and no carbon is apparent on the sample surface. No internal structure and rare porosity are seen inside the tungsten layer, a feature which is favourable to avoid delamination. The contamination of the constituting elements in the other layers is barely noticeable, showing that the coating build-up is controlled. Adhesion samples are prepared by gluing a screw on the tiles using a bicomponent Epoxy glue (STYCAST). Six CFC samples (no W-coating) are prepared, as well as the W-coated samples. Sequential gluing and pulling authorises 3 tests per coated sample. The samples are pulled using an INSTRON test bed equipped with a $25 \mathrm{kN}$ force sensor and the pulling speed was $0.5 \mathrm{~mm} /$ minute. A very good reproducibility of $11 \%$ is obtained on the 6 different CFC samples, showing that the mounting apparatus introduces few torque or misalignment. The adhesion stress is $13.5 \mathrm{MPa}$, which is approximately $30 \%$ of the CFC tensile strength (N11 from Safran), of the same order of the solidified glue (typical strength $50 \mathrm{MPa}$ ). Tests on the $\mathrm{W}$-coating are also reproducible, and give a mean value of 12.9 MPA which is $96 \%$ of the pure CFC samples. This very good result is confirmed by the observation of CFC fibers on the withdrawn screw, proving that the rupture occurs at least partly in the CFC material and not through delamination of the coating.

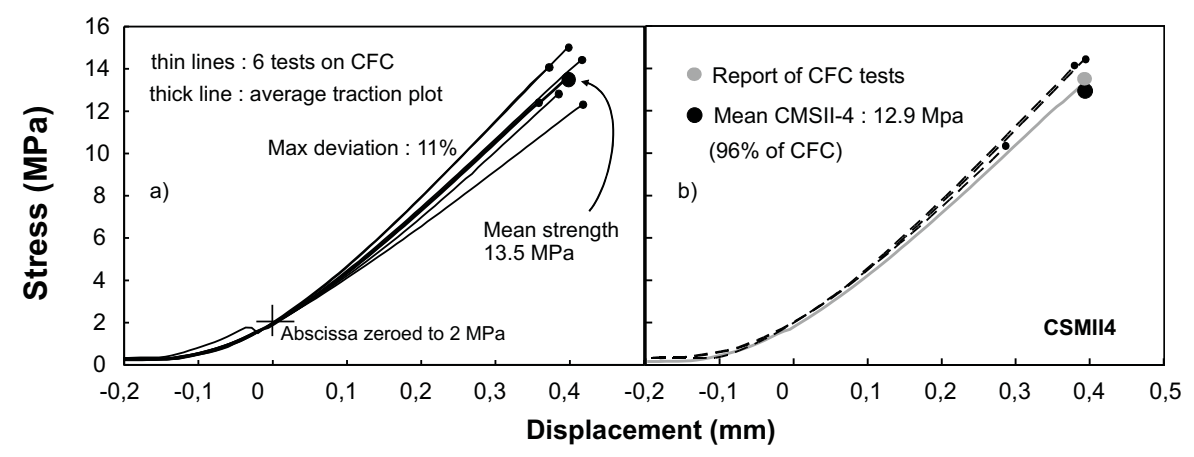

Figure 7 : Stress-displacement plots. a) for CFC tiles without W coating, b) for a tile with W coating. 


\section{CONCLUSION}

In the course of this work, three techniques to produce thick tungsten bonds to various substrates have been preliminarily investigated and partly characterised. All techniques deliver promising results and deserve in-depth investigations. The development of a reliable thick tungsten bond would however require a defined program, along with the collaboration of industrial manufacturers willing to acquire this technology. Due to the long time scale of this development, if such a program is to be established, it must probably be decoupled from the specific $R \& D$ accompanying ongoing manufacturing series.

\section{ACKNOWLEDGMENTS}

Parts of this work (sections 2 and 4), supported by the European Communities under the contract of Association between EURATOM and CEA, were carried out within the framework of the European Fusion Development Agreement. The views and opinions expressed herein do not necessarily reflect those of the European Commission.

\section{REFERENCES}

1 J. H. You and H. Bolt, J. Nucl. Mat., Volume 299, Issue 1, October 2001, Pp. 1-8

2 V. Barabash, M. Akiba, A. Cardella, I. Mazul, B. C. Odegard Jr, L. Plöchl, R. Tivey and G. Vieider, J. Nucl. Mat., Volumes 283-287, Part 2, December 2000, Pp. 1248-1252

3 M. Merola, M. Akiba, V. Barabash and I. Mazul, J. Nucl. Mat., Volumes 307311, Part 2, December 2002, Pp. 15241532

4 B. C. Odegard Jr., C. H. Cadden, R. D. Watson and K. T. Slattery, J. Nucl. Mat., Volumes 258-263, Part 1 , October 1998, Pp. 329-334
5 M. Missirlian, F. Escourbiac, M. Merola, I. Bobin-Vastra, J. Schlosser and A. Durocher, Fus. Eng. Des. , Volumes 75-79, November 2005, Pp. 435-440

6 A. Kurumada, Y. Imamura, Y. Tomota, T. Oku, Y. Kubota and N. Noda, J. Nucl. Mat., Volumes 313-316, March 2003, Pp. 245-249

7 I. Smid, M. Akiba, G. Vieider and L. Plöchl, J. Nucl. Mat., Volumes 258-263, Part 1, October 1998, Pp. 160-172

8. Jech D.E., Sepulveda J.L. and Traversone A.B., Patent US005993731A (Brush Wellman, Inc., U.S.A., 1999).

9 Ozer O, Missiaen JM, Pascal C, Lay S, Chaix JM, Mitteau R. Tungsten/Copper Functionally Graded Materials: Possible Applications and Processing through the Powder Metallurgy Route. MSF 2007;534-536:1569-72. https://doi.org/10.4028/www.scientific. net/msf.534-536.1569.

$10 \mathrm{~T}$. Hirai et al., R\&D on full tungsten divertor and beryllium wall for JET ITER-like Wall Project, Fusion Engineering and Design 82 (2007) 1839-1845

11 C.Ruset, E.Grigore, H.Maier, R.Neu, X.Li, H.Dong, R.Mitteau, X.Courtois, W coatings deposited on CFC tiles by Combined Magnetron Sputtering and Ion Implantation technique, to be published in physica scripta, 11th International Workshop on PlasmaFacing Materials and Components for Fusion Applications, October 10 - 12, 2006, Greifswald, Germany

12 C.P. Lungu, I. Mustata, G. Musa, A.M. Lungu, V. Zaroschi, K. Iwasaki, R. Tanaka, Y. Matsumura, I. Iwanaga, H. Tanaka et al., Surface and Coatings Technology, Volume 200, Issues 1-4, 1 October 2005, Pp. 399-402 